\title{
2018 Awards in the Journal of Plant Research
}

\author{
Kouki Hikosaka ${ }^{1}$
}

Published online: 6 August 2018

(c) The Botanical Society of Japan and Springer Japan KK, part of Springer Nature 2018

The Botanical Society of Japan honors excellence in publications of the Journal of Plant Research through the Best Paper Awards and the Most-Cited Paper Award every year. We are proud to announce this year's recipients.

\section{Best paper awards}

Kaori Takemura and Yuko T. Hanba in the Department of Applied Biology at the Kyoto Institute of Technology, Hiroyuki Kamachi and Ichirou Karahara in the Graduate School of Science and Engineering at the University of Toyama, Atsushi Kume in the Faculty of Agriculture at Kyushu University, and Tomomichi Fujita in the Faculty of Science at Hokkaido University have studied responses of growth and photosynthesis in bryophytes to hypergravity conditions (Takemura et al. 2017). In the evolutionary history of plants, bryophytes are the oldest plant groups to expand their habitats from aquatic to land environments. Effects of gravity on land may undoubtedly have induced alterations in the physiology, anatomy, and growth of bryophytes. The authors raised a model plant of moss, Physcomitrella patens, under a hypergravity environment using a custom-built centrifuge equipped with a lighting system. They found that plant growth is enhanced under a hypergravity environment and suggested that an increase in canopy-based plant numbers is effective to enhance the toughness of the population. Their finding contributes to deeper understanding of plant adaptation to gravity.

Kotaro T. Yamamoto, Masaaki K. Watahiki, Jun Matsuzaki, and Soichirou Satoh in the Faculty of Science at Hokkaido University and Hisayo Shimizu in the Graduate School of Life Science at Hokkaido University studied gravitropic response, which is one of the most fundamental growth

Kouki Hikosaka

hikosaka@m.tohoku.ac.jp

1 Graduate School of Life Sciences, Tohoku University, Aoba, Sendai 980-8578, Japan responses in plants (Yamamoto et al. 2017). They conducted an imaging analysis of the gravitropic response of etiolated Arabidopsis hypocotyls, using an IAA19 promoter fusion of destabilized luciferase as a probe. They found that the gravitropic response is composed of three successive elements that are each determined by separate principles: the first and second curving responses and a decurving response. Their finding contributes to deeper understanding of mechanisms and principles in gravitropic response in plants.

\section{Most-cited paper award}

Yasutaka Chiba, Shinya Miyakawa, Tomokazu Koshiba, Yuji Kamiya, and Mitsunori Seo in the Department of Biological Sciences at Tokyo Metropolitan University and Takafumi Shimizu, Yuri Kanno, and Yuji Kamiya in the RIKEN Center for Sustainable Resource Science studied NRT1/PTR FAMILY (NPF) proteins, which were originally identified as nitrate or di/tri-peptide transporters (Chiba et al. 2015). The authors developed modified yeast two-hybrid systems with receptor complexes for GA and jasmonoyl-isoleucine (JAIle), to detect GA and JA-Ile transport activities of proteins expressed in the yeast cells. They found that some NPFs have hormone transport activities. This paper has been cited in 40 papers since 2015 .

\section{Open access}

The Botanical Society of Japan sponsored the conversion of seven papers originally published without open access to open access in 2018. Two of those are awardees of the best paper award mentioned in the section above (Takemura et al. 2017; Yamamoto et al. 2017). Five papers are reviews published in the category of "Current Topics in Plant Research" (Inada 2017; Inoue et al. 2018; Kobayashi 2016; Kotake et al. 2016; Kume 2017). We hope that the papers will now reach a wider audience and contribute further to the progress of plant science. 
Kouki Hikosaka

Editor-in-Chief, Journal of Plant Research

\section{References}

Chiba Y, Shimizu T, Miyakawa S, Kanno ZY, Koshiba T, Kamiya Y, Seo M (2015) Identification of Arabidopsis thaliana NRT1/PTR FAMILY (NPF) proteins capable of transporting plant hormones. J Plant Res 128:679-686

Inada N (2017) Plant actin depolymerizing factor: actin microfilament disassembly and more. J Plant Res 130:227-238

Inoue K, Araki T, Endo M (2018) Circadian clock during plant development. J Plant Res 131:59-66

Kobayashi K (2016) Role of membrane glycerolipids in photosynthesis, thylakoid biogenesis and chloroplast development. J Plant Res 129:565-580
Kotake T, Yamanashi Y, Imaizumi C, Tsumuraya Y (2016) Metabolism of L-arabinose in plants. J Plant Res 129:781-792

Kume A (2017) Importance of the green color, absorption gradient, and spectral absorption of chloroplasts for the radiative energy balance of leaves. J Plant Res 130:501-514

Takemura K, Kamachi H, Kume A, Fujita T, Karahara I, Hanba YT (2017) A hypergravity environment increases chloroplast size, photosynthesis, and plant growth in the moss Physcomitrella patens. J Plant Res 130:181-192

Yamamoto KT, Watahiki MK, Matsuzaki J, Satoh S, Shimizu H (2017) Space-time analysis of gravitropism in etiolated Arabidopsis hypocotyls using bioluminescence imaging of the IAA19 promoter fusion with a destabilized luciferase reporter. J Plant Res 130:765-777 